\title{
The science case of the FRS Ion Catcher for FAIR Phase-0
}

\begin{abstract}
Wolfgang R. Plaß $ß^{1,2}$ D . Timo Dickel ${ }^{1,2} \cdot$ Israel Mardor $^{3,4}$. Stephane Pietri ${ }^{2}$. Hans Geissel ${ }^{1,2}$. Christoph Scheidenberger ${ }^{1,2}$ - Daler Amanbayev ${ }^{1}$. Samuel Ayet San Andrés ${ }^{1,2}$. Juha Äystö ${ }^{5,6}$. Dimiter L. Balabanski ${ }^{7}$. Sönke Beck ${ }^{1,2}$. Julian Bergmann ${ }^{1}$. Volha Charviakova ${ }^{8}$. Paul Constantin ${ }^{7}$. Tommi Eronen ${ }^{6}$. Tuomas Grahn ${ }^{6}$. Florian Greiner ${ }^{1}$ • Emma Haettner ${ }^{2}$. Christine Hornung $^{1}$. Jean-Paul Hucka ${ }^{2}$. Ari Jokinen ${ }^{6}$ - Anu Kankainen ${ }^{6}$. Ivan Miskun ${ }^{1}$ - Iain D. Moore ${ }^{6}$. Alexander Pikthtelev ${ }^{9}$. Ilkka Pohjalainen ${ }^{6}$. Sivaji Purushothman ${ }^{2}$. Zygmunt Patyk ${ }^{8}$. Moritz P. Reiter ${ }^{1,2,10}$. Ann-Kathrin Rink ${ }^{1}$. Sami Rinta-Antila ${ }^{6}$ - Anamaria Spataru 7,11 . Helmut Weick ${ }^{2}$. John S. Winfield ${ }^{2}$. Mikhail I. Yavor ${ }^{12}$ for the Super-FRS Experiment Collaboration
\end{abstract}

Published online: 24 June 2019

(C) The Author(s) 2019

\begin{abstract}
The FRS Ion Catcher at GSI enables precision experiments with thermalized projectile and fission fragments. At the same time it serves as a test facility for the Low-Energy Branch of the Super-FRS at FAIR. The FRS Ion Catcher has been commissioned and its performance has been characterized in five experiments with ${ }^{238} \mathrm{U}$ and ${ }^{124} \mathrm{Xe}$ projectile and fission fragments produced at energies in the range from 300 to $1000 \mathrm{MeV} / \mathrm{u}$. High and almost element-independent efficiencies for the thermalization of short-lived nuclides produced at relativistic energies have been obtained. High-accuracy mass measurements of more than 30 projectile and fission fragments have been performed with a multiple-reflection time-offlight mass spectrometer (MR-TOF-MS) at mass resolving powers of up to 410,000, with production cross sections down to the microbarn-level, and at rates down to a few ions per hour. The versatility of the MR-TOF-MS for isomer research has been demonstrated by the measurement of various isomers, determination of excitation energies and the production of a pure isomeric beam. Recently, several instrumental upgrades have been implemented at the FRS Ion Catcher. New experiments will be carried out during FAIR Phase-0 at GSI, including direct mass measurements of neutron-deficient nuclides below ${ }^{100} \mathrm{Sn}$ and neutronrich nuclides below ${ }^{208} \mathrm{~Pb}$, measurement of $\beta$-delayed neutron emission probabilities and reaction studies with multi-nucleon transfer.
\end{abstract}

This article is part of the Topical Collection on Proceedings of the 7th International Conference on Trapped Charged Particles and Fundamental Physics (TCP 2018), Traverse City, Michigan, USA, 30 September-5 October 2018

Edited by Ryan Ringle, Stefan Schwarz, Alain Lapierre, Oscar Naviliat-Cuncic, Jaideep Singh and Georg Bollen

Wolfgang R. Plaß

Wolfgang.R.Plass@exp2.physik.uni-giessen.de

Extended author information available on the last page of the article. 
Keywords Mass measurements · MR-TOF-MS · Exotic nuclides · Nuclear reactions · Beta-delayed neutron emission

\section{Introduction}

At the FRS Ion Catcher (FRS-IC) [1] precision experiments with thermalized exotic nuclei can be performed. The fragments are produced by projectile fragmentation or fission at relativistic energies, separated in-flight and energy-bunched in the fragment separator FRS [2]. With the FRS-IC (Fig. 1) these fragments are then slowed down and thermalized in a cryogenic stopping cell (CSC) [3]. A multiple-reflection time-of-flight mass spectrometer (MR-TOF-MS) is used to perform direct mass measurements and to provide an isobarically and isomerically clean beam for further experiments, such as mass-selected decay spectroscopy $[4,5]$. A versatile RF quadrupole (RFQ) beam line guides the ions from the CSC to the MR-TOF-MS, provides differential pumping, ion identification and mass separation and includes reference ion sources. The FRS-IC serves as a test facility for the Low-Energy Branch (LEB) of the Super-FRS and already now enables a variety of new precision experiments in FAIR Phase-0.

\section{Recent results and instrumental upgrades}

The FRS-IC has been commissioned and its performance has been characterized in five experiments with ${ }^{238} \mathrm{U}$ and ${ }^{124} \mathrm{Xe}$ projectile and fission fragments produced at energies in the range from 300 to $1000 \mathrm{MeV} / \mathrm{u}$. For the first time, a stopping cell for exotic nuclei was operated on-line at cryogenic temperatures. Using a gas density almost three times higher than ever reached before for a stopping cell with RF ion repelling structures, various shortlived nuclei were thermalized and extracted $[1,6,7]$. Ion survival and extraction efficiencies of more than $60 \%$ have been achieved for different elements, including noble gases (e.g. $\mathrm{Rn}$ ) as well as highly chemically reactive elements (e.g. Th), see Fig. 2. This demonstrates the universality of the method. A combined ion stopping, survival and extraction efficiency of up to $30 \%$ and an extraction time of about $25 \mathrm{~ms}$ have been measured. Furthermore, the rate capability of the CSC has been investigated [8].

Direct mass measurements of several short-lived nuclides with half-lives down to $2 \mathrm{~ms}$, with production cross sections down to the microbarn-level, at rates down to a few ions per hour, and for as few as 11 detected ions per species have been performed with the MRTOF-MS [9-14]. Mass resolving powers of up to 410,000 have been achieved, the highest resolving power obtained hitherto online with an MR-TOF-MS. In total for more than 30 short-lived nuclei the ground state masses have been determined and various isomeric states have been measured. A data evaluation procedure for MR-TOF-MS data has been developed that allows achieving high mass measurement accuracies even for a few detected ions only and for overlapping mass peaks, e.g. due to low-lying isomers. The error budget of the measurement with the MR-TOF-MS has been investigated; relative mass uncertainties down to $6 \times 10^{-8}$ have been achieved. Furthermore, the FRS-IC has been shown to be an ideal setup to search for long-lived isomers with half-lives longer than a few microseconds, because it is universal, i.e. independent of element and decay mode of the nuclides, faster than most other mass spectrometry techniques for exotic nuclides, very sensitive (non-scanning), has a high mass resolving power, a large dynamic range and is broadband in nature, i.e. it can measure all isobars and isomers of several mass units in parallel. Moreover, the isomers can 


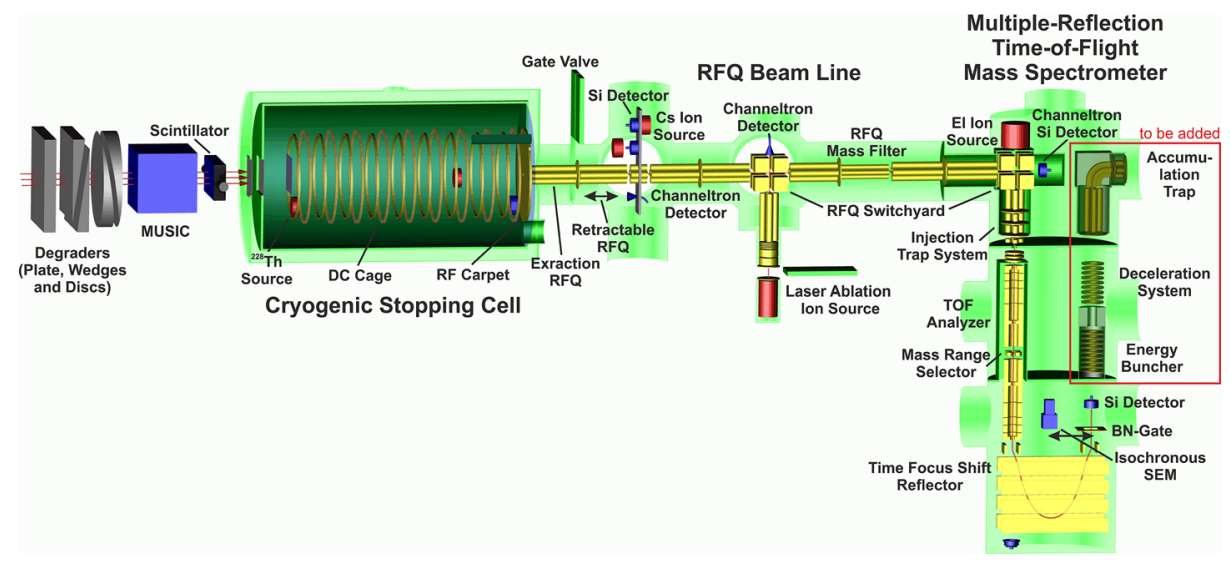

Fig. 1 Schematic view of the setup of the FRS Ion Catcher at the final focal plane of the FRS. The beam enters the setup from the FRS from the left and passes the detectors for the particle identification in the FRS and a variable degrader, which is used to adjust the range of the ions. The ions are then thermalized in the CSC, extracted and transmitted through the RFQ beam line to the MR-TOF-MS

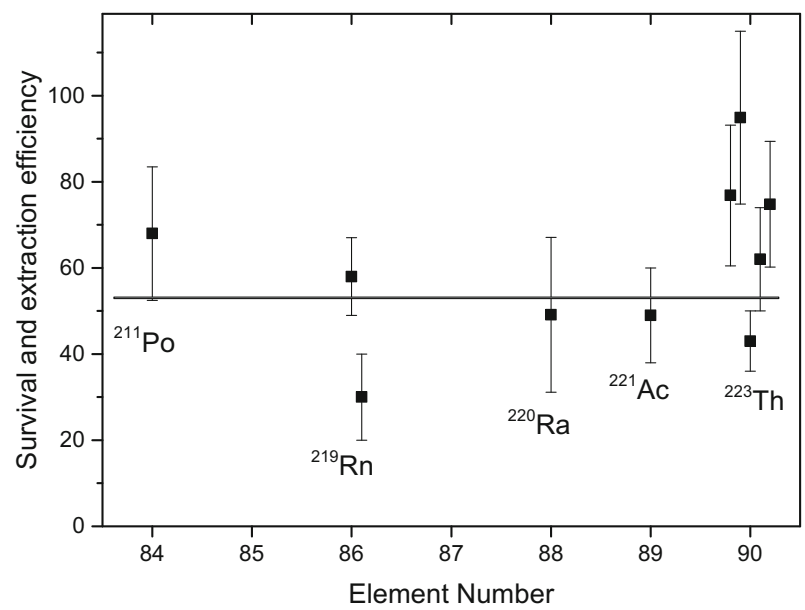

Fig. 2 Measured ion survival and extraction efficiencies with the cryogenic stopping cell of the FRS-IC for different elements produced by in-flight fragmentation of ${ }^{238} \mathrm{U}$ at $1 \mathrm{GeV} / \mathrm{u}$

be spatially separated from the ground state and an isomerically clean beam can be provided to experiments, e.g. for decay spectroscopy [5].

Recently, the FRS-IC has been upgraded in several aspects. In particular, (i) in the CSC the electrode structure and the connections have been revised to increase the operational reliability and to allow for higher extraction field strengths and thus shorter extraction times. (ii) A new pre-pump allows operation of the turbo-molecular pumps on the RFQ beam line, which serves as first differential pumping stage behind the CSC, at higher pressures. (iii) An extension of the RFQ beam line has been developed and commissioned, which includes a dedicated RFQ mass filter and a laser ablation ion source for providing ions for mass 
calibration [15]. The upgrades (ii) and (iii) in combination allow the operation of the CSC with heavy ions at higher areal densities of up to $10 \mathrm{mg} / \mathrm{cm}^{2}$ [16].

\section{Science case of the FRS Ion Catcher for FAIR Phase-0}

After the successful commissioning of the FRS-IC and its upgrade, dedicated physics experiments will be performed in the coming years. Four experiment proposals for experiments in FAIR Phase-0 have been submitted to the program advisory committee of GSI and approved. They are discussed in the following.

\subsection{Direct mass measurements of neutron-deficient nuclides below ${ }^{100} \mathrm{Sn}$}

The nuclides on or in the vicinity of the $N=Z$ line below ${ }^{100} \mathrm{Sn}$ are of high interest because of a multitude of physics questions, among them isospin symmetry, mirror nuclei, and the Wigner energy in $N=Z$ nuclei $[17,18]$, the region of nuclear deformation in the vicinity of ${ }^{80} \mathrm{Zr}$ [19], the high-spin isomer in ${ }^{94} \mathrm{Ag}$ [20, 21], and the $r p$ nucleosynthesis process [22]. Mass measurements of $N=Z$ and $N=Z+1$ nuclides in the element range from Y to Ag will be performed with the FRS-IC; for many of these nuclides they will provide experimental mass values for the first time (Fig. 3) [23]. The nuclei will be produced by projectile fragmentation of ${ }^{124} \mathrm{Xe}$ and ${ }^{107} \mathrm{Ag}$. Since the elements in the range from $\mathrm{Zr}$ to $\mathrm{Rh}$ are refractive, these nuclei can be ideally produced and separated in-flight.

The use of very thick targets $\left(\gg 4 \mathrm{~g} / \mathrm{cm}^{2} ; d / R>0.5\right.$, where $d$ is the target thickness and $R$ the range of the ions in matter) will be investigated, which should increase yields by multi-step fragmentation reactions and enable access to more exotic nuclides at the FRS-IC and the LEB of the Super-FRS, making optimum use of the high primary beam energies.

Furthermore, in the experiment, the recent instrumental upgrades of the FRS-IC and developments for the CSC of the LEB of the Super-FRS will be commissioned online, including the pumping and $\mathrm{He}$ recycling unit and the gas purification techniques to be used with the CSC of the Super-FRS as well as the controlled manipulation of charge states of the thermalized ions in order to further increase the overall efficiency [24].

\subsection{Direct mass measurements of neutron-rich nuclides below ${ }^{208} \mathrm{~Pb}$}

The region along the $N=126$ line below ${ }^{208} \mathrm{~Pb}$ has been identified as a region of key interest for the NUSTAR collaboration at GSI/FAIR; generally it is of high interest for nuclear structure studies and for nuclear astrophysics applications. An experiment will be performed with the FRS to extend the frontiers of known isotopes in this region and to obtain nuclear structure data on gross properties [26]. New neutron-rich isotopes will be produced and identified in the element range between $\mathrm{Tb}$ and Re using projectile fragmentation of ${ }^{208} \mathrm{~Pb}$ and their production cross sections will be measured. At the same time, masses and life times will be determined (Fig. 4). The beam time will thus be used very efficiently by the simultaneous use of several detection systems and the multiple use of the same ions. The mass measurements will be performed employing the FRS-IC; the new mass values will be highly relevant for the third peak of the $r$ process. 


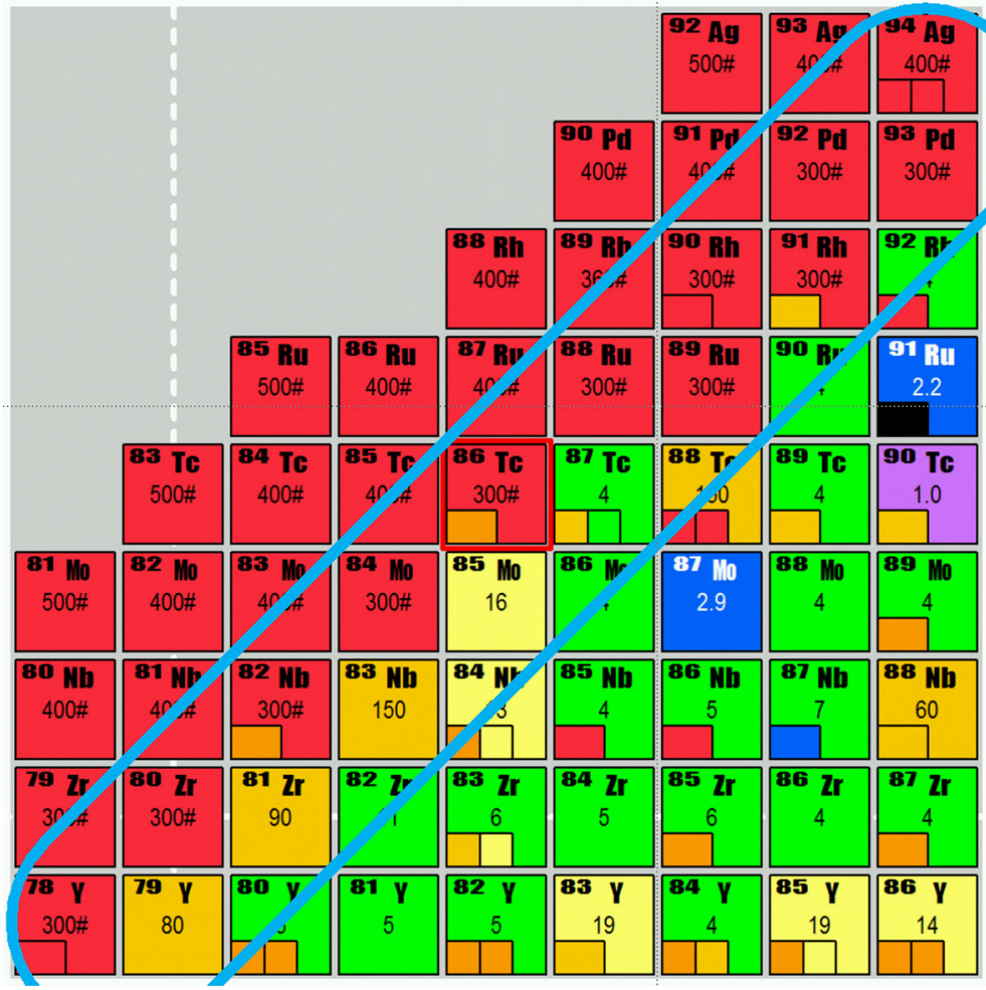

Fig. 3 Section of the chart of the nuclides showing the region of interest below ${ }^{100} \mathrm{Sn}$ and the uncertainty of mass values in the elemental range from $\mathrm{Y}$ to $\mathrm{Ag}$ (in $\mathrm{keV}$ ). For nuclides shown in red the mass has not been measured yet [25]. Left: Expected charge states of the ions extracted from the CSC and range of refractory elements in this region

\subsection{Measurement of $\beta$-delayed neutron emission probabilities}

Measured probabilities for $\beta$-delayed neutron emission provide important input for the understanding of the astrophysical $r$ process, for nuclear structure models, and for control of nuclear reactors [27]. Data for $\beta$-delayed neutron emission probabilities are scarce, especially for multiple-neutron emission [28]. Therefore, there are several world-wide campaigns to bridge this data gap, mostly by counting $\beta$-delayed emission $\mathrm{n}-\beta$ coincidence events [29]. Other proposed methods count the recoil nuclides via gamma spectra [30], time-of-flight [31] or particle detectors in a storage ring [32].

It has been proposed to use a series of stopping cells and MR-TOF-MSs for measurement of $\beta$-delayed neutron emission probabilities [33]. We have employed a similar method, however with only one stopping cell and MR-TOF-MS, to perform a proof-of-principle measurement of branching ratios for $\beta$ - and $\gamma$-decays with the FRS-IC [14]. In FAIR-Phase0 , this method will be used for the measurement of $\beta$-delayed neutron emission probabilities [34] (Fig. 5). The nuclides of interest are produced and separated with the FRS, thermalized and stored in the CSC for periods long enough for them to decay. After extraction from the $\mathrm{CSC}$, the precursor and recoil isotopes are identified and counted using the MR-TOF-MS. The masses of the precursor and all recoils are measured simultaneously with an accuracy 


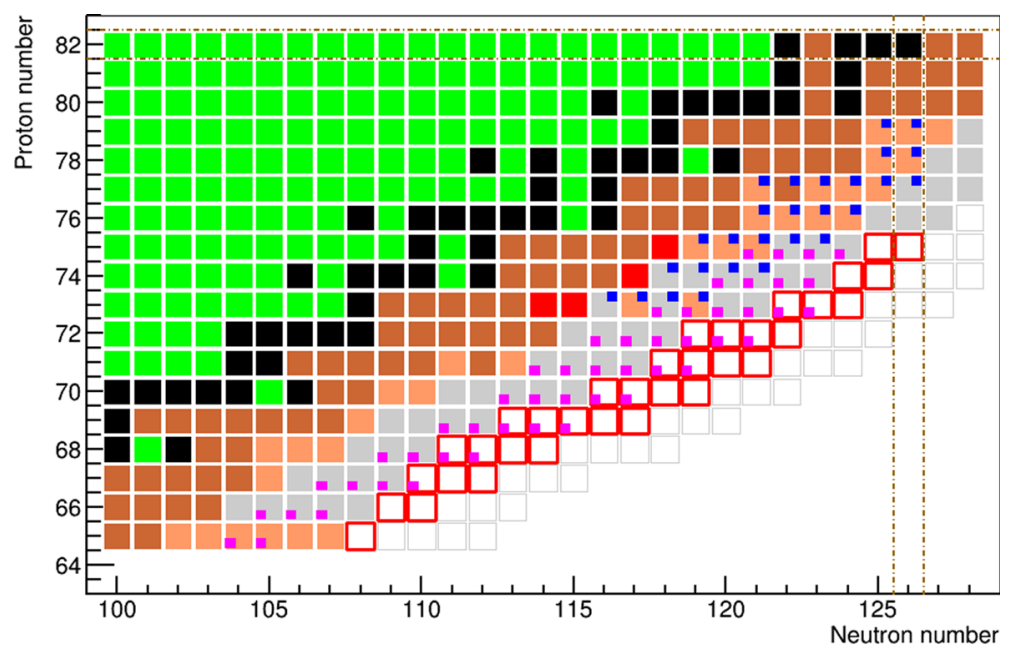

Fig. 4 Section of the chart of the nuclides below ${ }^{208} \mathrm{~Pb}$ indicating the nuclides to be measured. Black: stable nuclei; green: neutron deficient nuclei; dark brown: isotopes with known half-lives and masses; salmon: nuclei with known half-lives; red: nuclei with known mass; grey: already discovered isotopes; red borders: new isotopes from this proposal; pink squares: proposed half-live measurements; blue squares: proposed mass measurements

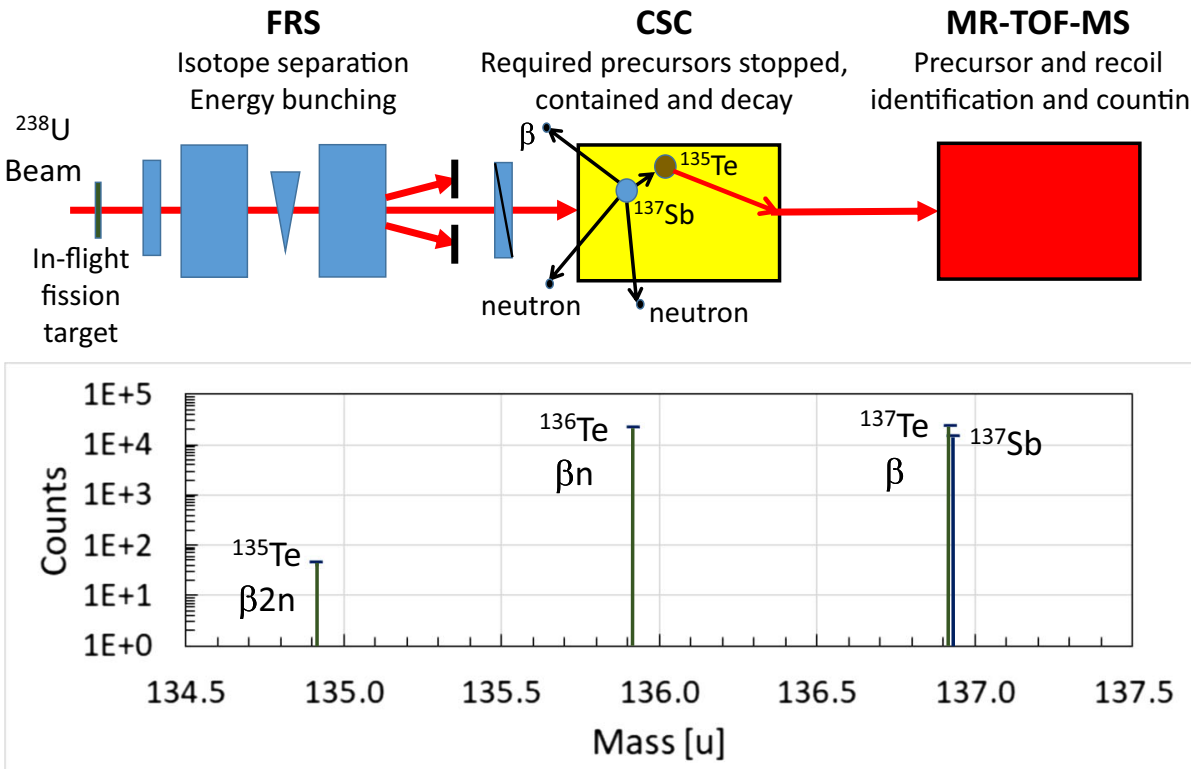

Fig. 5 Upper panel: Scheme of method proposed to measure probabilities for $\beta$-delayed neutron emission. A $\beta_{2 n}$ decay of ${ }^{137} \mathrm{Sb}$ in the CSC is depicted. Lower panel: Expected spectrum in the MR-TOF-MS, when storing the precursors in the CSC for two ${ }^{137} \mathrm{Sb}$ half-lives 
that enables their unambiguous identification. Ratios between the measured abundances of the recoil isotopes infer the $\beta$-delayed emission probabilities for one and multiple neutrons. The method has the same efficiency regardless of the number of neutrons, is independent of nuclear models, has practically no background, and is sensitive to isomers. First measurements will be performed for ${ }^{135-137} \mathrm{Sb}$ and ${ }^{142} \mathrm{I}$, the heaviest and highest $Z \beta_{2 \text { n }}$ precursor measured yet, setting the stage for a survey of $\beta$-delayed neutron emission, including the $N \sim 126$ region.

\subsection{Reaction studies with multi-nucleon transfer}

Experiments with radioactive ion beams at Coulomb barrier energies represent a new field for reaction studies, which will contribute to a better understanding of multi-nucleon transfer (MNT), deep inelastic, fusion-fission and complete fusion reactions. One of the main goals of these experiments is to access new neutron-rich heavy and super-heavy isotopes, not reachable in fission or fragmentation reactions [35]. Slowed-down rare ion beams of all chemical elements, including refractory elements, independent of their chemical properties, are available at the FRS and Super-FRS. Their optimum use requires a new philosophy for experiments and instrumentation [36]. For instance, MNT reactions inside the CSC of the FRS-IC are a unique and novel method that is very sensitive, clean, universal and has a high angular acceptance for the reaction products [37]. It is planned to mount several different targets for MNT reactions on a wheel, which will be installed in the stopping volume of the CSC. The identification and quantification of the reaction products will be performed by the MR-TOF-MS. This approach will pave the way for broad-range measurements with primary and secondary beams.

In a first experiment, MNT reaction studies will be performed with a $500 \mathrm{MeV} / \mathrm{u}{ }^{238} \mathrm{U}$ primary beam slowed-down to Coulomb barrier energies impinging on different targets. It is expected that more than 150 cross sections and 15 new masses of neutron-rich nuclides can be measured (Fig. 6). The cross sections will be used to benchmark reaction models. The nuclides to be measured are important for the rare earth abundance peak in the $r$ process as well as for the understanding the properties of fissioning nuclei involved in the $r$ process.

\section{Summary and outlook}

The FRS Ion Catcher enables precision experiments with thermalized exotic nuclei. It has been commissioned in five experiments with ${ }^{238} \mathrm{U}$ and ${ }^{124} \mathrm{Xe}$ projectile and fission fragments. The setup has high and almost element-independent efficiencies. High-accuracy mass measurements of exotic nuclei in ground and low-lying isomeric states can be performed even with a few detected ions only. More than 30 short-lived ground state masses have been measured with relative mass uncertainties down to $6 \times 10^{-8}$. The excitation energies of various isomers and isomeric ratios have been determined, and, for the first time, an isomeric beam was prepared using an MR-TOF-MS. The unique combination of performance parameters make the FRS Ion Catcher the system of choice for measuring the masses of very exotic nuclei and for the search for new long-lived isomeric states. This is the basis for a broad scientific program in FAIR Phase-0, which includes mass measurements of $N=Z$ nuclides below ${ }^{100} \mathrm{Sn}$ and nuclides for the third peak of the $r$ process, measurements of beta-delayed neutron emission probabilities, and the investigation of multi-nucleon transfer reactions. 


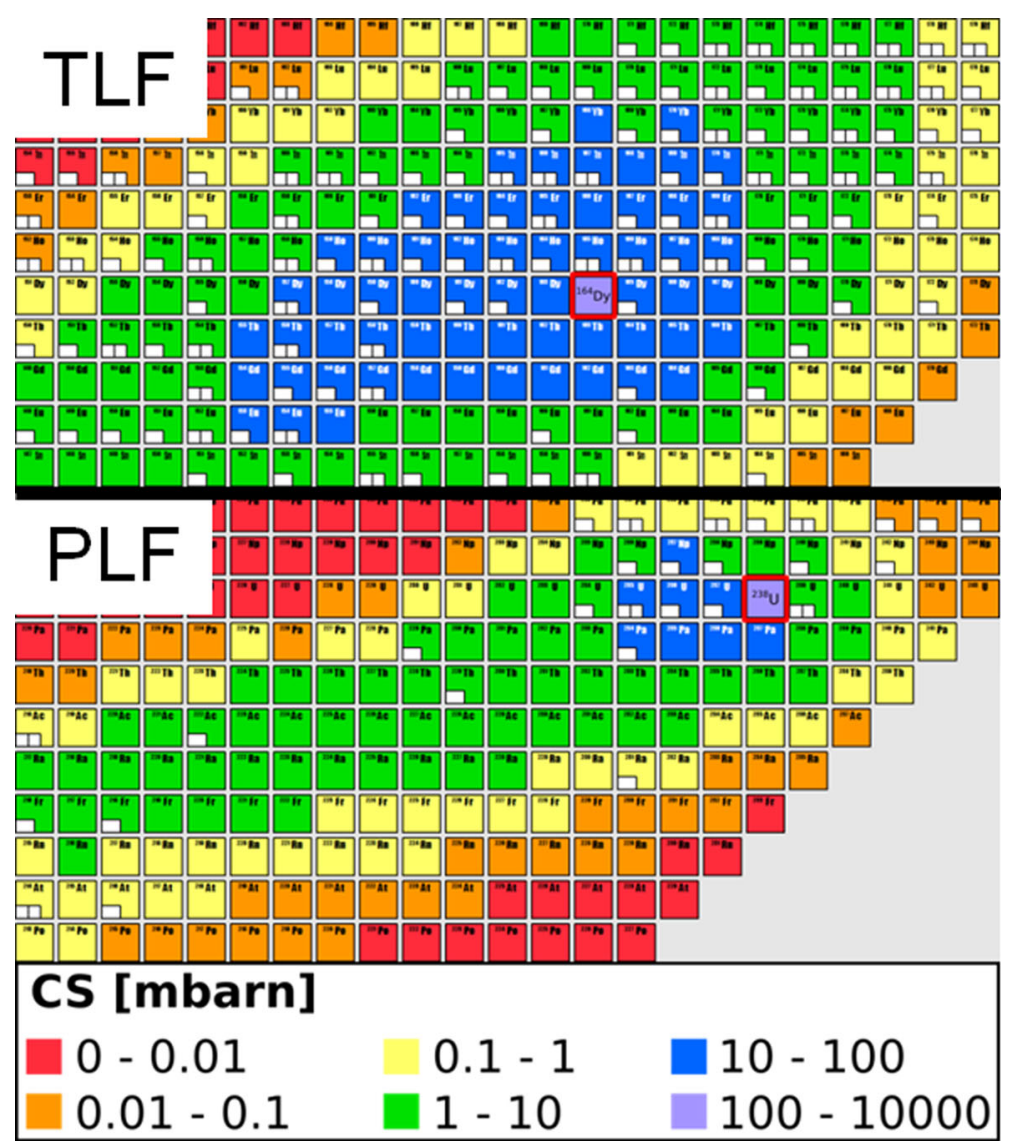

Fig. 6 Calculated production cross-sections [38] for ${ }^{238} \mathrm{U}$ on ${ }^{164} \mathrm{Dy}$. Upper panel: target-like fragments (TLF); lower panel: projectile-like fragments (PLF). The estimated sensitivity limit will be about 0.1 mbarn

Based on the experience with the FRS Ion Catcher, the development of the cryogenic stopping cell for the Low Energy Branch of the Super-FRS is underway [24, 39]; it will feature even higher stopping and thermalization efficiencies, shorter extraction times and higher rate capability.

Acknowledgments This work was supported by the German Federal Ministry for Education and Research (BMBF) under contracts no. 05P15RGFN1 and 05P19RGFN1, by Justus-Liebig-Universität Gießen and GSI under the JLU-GSI strategic Helmholtzpartnership agreement, by HGS-HIRe, by the Hessian Ministry for Science and Art (HMWK) through the LOEWE Center HICforFAIR, and by the European Union's Horizon 2020 research and innovation programme contract no. 654002 via the JRA SATNURSE. DLB, PC and AS are supported by Extreme Light Infrastructure Nuclear Physics (ELI-NP) Phase II, a project co-financed by the Romanian Government and the European Union through the European Regional Development Fund - the Competitiveness Operational Programme (1/07.07.2016, COP, ID 1334). 
Open Access This article is distributed under the terms of the Creative Commons Attribution 4.0 International License (http://creativecommons.org/licenses/by/4.0/), which permits unrestricted use, distribution, and reproduction in any medium, provided you give appropriate credit to the original author(s) and the source, provide a link to the Creative Commons license, and indicate if changes were made.

\section{References}

1. Plaß, W.R., et al.: The FRS Ion Catcher - A facility for high-precision experiments with stopped projectile and fission fragments. Nucl. Instrum. Methods B 317, 457-462 (2013)

2. Geissel, H. et al.: The GSI projectile fragment separator (FRS): a versatile magnetic system for relativistic heavy ions. Nucl. Instrum. Methods B 70, 457-462 (1992)

3. Ranjan, M. et al.: Design, construction and cooling system performance of a prototype cryogenic stopping cell for the Super-FRS at FAIR. Nucl. Instrum. Methods A 770, 87-97 (2015)

4. Dickel, T. et al:: A high-performance multiple-reflection time-of-flight mass spectrometer and isobar separator for the research with exotic nuclei. Nucl. Instrum. Methods A 777, 172-188 (2015)

5. Dickel, T. et al.: First spatial separation of a heavy ion isomeric beam with a multiple-reflection time-offlight mass spectrometer. Phys. Lett. B 744, 137-141 (2015)

6. Purushotaman, S. et al.: First experimental results of a cryogenic stopping cell with short-lived, heavy uranium fragments produced at $1000 \mathrm{MeV} / \mathrm{u}$. EPL 104, 4 (2013)

7. Reiter, M.P.: Pilot Experiments with Relativistic Uranium Projectile and Fission Fragments Thermalized in a Cryogenic Gas-Filled Stopping Cell, PhD Thesis, Justus Liebig University Gießen (2015)

8. Reiter, M.P. et al.: Rate capability of a cryogenic stopping cell for uranium projectile fragments produced at $1000 \mathrm{MeV} / \mathrm{u}$. Nucl. Instrum. Methods B 376, 240-245 (2016)

9. Purushotaman, S. et al.: Hyper-EMG: A new probability distribution function composed of Exponentially Modified Gaussian distributions to analyze asymmetric peak shapes in high-resolution time-of-flight mass spectrometry. Int. J. Mass Spectrom. 421, 245-254 (2017)

10. Rink, A.-K.: Mass and Life-time Measurement of the $1.7 \mathrm{~ms}{ }^{215}$ Po Isotope - A Crucial Test of the Novel Concept of the Cryogenic Ion Catcher for the Super FRS at GSI-FAIR, PhD Thesis, Justus Liebig University Gießen (2017)

11. Ayet San Andres, S.: Developments for multiple-reflection time-of-flight mass spectrometers and their application to high-resolution accurate mass measurements of short-lived exotic nuclei, $\mathrm{PhD}$ Thesis, Justus Liebig University Gießen (2018)

12. Hornung, C.: High-resolution Experiments with the Multiple-Reflection Time-of-Flight Mass Spectrometer at the Fragment Separator FRS, PhD Thesis, Justus Liebig University Gießen (2018)

13. Ayet San Andres, S. et al.: High-resolution, accurate multiple-reflection time-of-flight mass spectrometry for short-lived, exotic nuclei of a few events in their ground and low-lying isomeric states. Phys. Rev. C 99, 064313 (2019)

14. Miskun, I. et al.: A Novel Method for the Measurement of Half-Lives and Decay Branching Ratios of Exotic Nuclei, submitted for publication; arXiv:1902.11195

15. Hornung, C. et al.: An upgrade to the RFQ beam line of the FRS Ion Catcher. GSI-FAIR Sci. Rep. 2017, 116 (2018). https://doi.org/10.15120/GR-2018-1

16. Miskun, I.: PhD Thesis, Justus Liebig University Gießen, in preparation

17. Van Isacker, P. et al.: Test of Wigner's Spin-Isospin Symmetry from Double Binding Energy Differences. Phys. Rev. Lett. 74, 4607-4610 (1995)

18. Lalleman, A.S. et al.: Mass measurements of exotic nuclei around $N=Z=40$ with CSS2. Hyperfine Interact 132, 315-322 (2001)

19. Lister, C.J. et al.: Gamma radiation from the $\mathrm{N}=\mathrm{Z}$ nucleus ${ }^{80} \mathrm{Zr}$. Phys. Rev. Lett. 59, 1270 (1987)

20. Mukha, I. et al.: Proton-proton correlations observed in two-proton radioactivity of ${ }^{94} \mathrm{Ag}$. Nature 439, 298-302 (2006)

21. Kankainen, A. et al.: Mass measurements and implications for the energy of the High-Spin isomer in ${ }^{94}$ Ag. Phys. Rev. Lett. 101, 142503 (2008)

22. Schatz, H. et al.: rp-process nucleosynthesis at extreme temperature and density conditions. Phys. Rep. 294, 167-263 (1998)

23. Plaß, W.R. et al.: Detector tests with the prototype CSC for the Super-FRS and direct mass measurements of neutron-deficient nuclides below ${ }^{100} \mathrm{Sn}$, Proposal to the G-PAC for FAIR Phase-0 Experiments, GSI Darmstadt. GSI-FAIR Sci. Rep. 2017, 115 (2018). https://doi.org/10.15120/GR-2018-1 
24. Dickel, T. et al.: Conceptional design of a novel next-generation cryogenic stopping cell for the LowEnergy Branch of the Super-FRS. Nucl. Instrum. Methods B 376, 216-220 (2016)

25. Huang, W.J. et al.: The AME2016 atomic mass evaluation. Chin. Phys. C 41, 3 (2017)

26. Pietri, S. et al.: Search for new neutron-rich isotopes and exploratory studies in the element range from terbium to rhenium, Proposal to the G-PAC for FAIR Phase-0 Experiments, GSI Darmstadt. 2017. GSIFAIR Sci. Rep. 2017, 99 (2018). https://doi.org/10.15120/GR-2018-1

27. Mumpower, M.R. et al.: Neutron- $\gamma$ competition for $\beta$-delayed neutron emission. Phys. Rev. C 94, 064317 (2017)

28. Dillmann, I. et al.: Recent activities for $\beta$-decay half-lives and $\beta$-delayed neutron emission of very neutron-rich isotopes. AIP Conf. Proc. 1594, 332 (2014)

29. Tarifeño-Saldivia, A. et al.: Conceptual design of a hybrid neutron-gamma detector for study of $\beta$ delayed neutrons at the RIB facility of RIKEN. J. Instrum. 12, P04006 (2017)

30. Winger, J.A. et al.: Large $\beta$-Delayed Neutron Emission Probabilities in the ${ }^{78} \mathrm{Ni}$ Region. Phys. Rev. Lett. 102, 142502 (2009)

31. Yee, R.M. et al.: $\beta$-Delayed Neutron Spectroscopy Using Trapped Radioactive Ions. Phys. Rev. Lett. 110, 092501 (2013)

32. Evdokimov, A. et al.: An alternative approach to measure $\beta$-delayed neutron emission. Proceedings of Science (NIC XII) 115 (2012)

33. Miyatake, H. et al.: AIP Conf. Proc. 1947, 020018 (2018)

34. Mardor, I. et al.: A Novel Method for Measuring $\beta$-Delayed Neutron Emission, Proposal to the G-PAC for FAIR Phase-0 Experiments, GSI Darmstadt. GSI-FAIR Sci. Rep. 2017, 114 (2018). https://doi.org/10.15120/GR-2018-1

35. Zagrebaev, V. et al.: Production of new heavy isotopes in low-energy multinucleon transfer reactions. Phys. Rev. Lett. 101, 122701 (2008)

36. Münzenberg, G. et al.: SHE Research on the way to NUSTAR and FAIR. In: Penionzhkevich, Y., Soblev, Y. (eds.) Exotic Nuclei: EXON-2014 - Proceedings of International Symposium, pp. 541-550. World Scientific Publishing (2015)

37. Dickel, T. et al.: Reaction studies with the FRS Ion Catcher: A novel approach and universal method for the production, identification of and experiments with unstable isotopes produced in multi-nucleon transfer reactions with stable and unstable beams, Proposal to the G-PAC for FAIR Phase-0 Experiments, GSI Darmstadt. GSI-FAIR Sci. Rep. 2017, 112 (2018). https://doi.org/10.15120/GR-2018-1

38. Karpov, A.V. et al.: Modeling near-barrier collisions of heavy ions based on a Langevin-type approach. Phys. Rev. C 96, 024618 (2017)

39. Amanbayev, D. et al.: Development of the stopping cell for the Low-Energy Branch of the Super-FRS. GSI-FAIR Sci. Rep. 2017, 117 (2018). https://doi.org/10.15120/GR-2018-1

Publisher's note Springer Nature remains neutral with regard to jurisdictional claims in published maps and institutional affiliations. 


\section{Affiliations}

Wolfgang R. Plaß $ß^{1,2}$ (D) Timo Dickel ${ }^{1,2}$ - Israel Mardor ${ }^{3,4}$. Stephane Pietri ${ }^{2}$. Hans Geissel ${ }^{1,2}$. Christoph Scheidenberger ${ }^{1,2}$. Daler Amanbayev ${ }^{1}$. Samuel Ayet San Andrés ${ }^{1,2}$. Juha Äystö ${ }^{5,6}$. Dimiter L. Balabanski ${ }^{7}$. Sönke Beck ${ }^{1,2}$. Julian Bergmann ${ }^{1}$. Volha Charviakova ${ }^{8}$. Paul Constantin ${ }^{7}$. Tommi Eronen $^{6}$. Tuomas Grahn ${ }^{6}$. Florian Greiner ${ }^{1}$. Emma Haettner ${ }^{2}$. Christine Hornung $^{1}$. Jean-Paul Hucka ${ }^{2}$. Ari Jokinen ${ }^{6}$ - Anu Kankainen ${ }^{6}$. Ivan Miskun $^{1}$ - lain D. Moore ${ }^{6}$ - Alexander Pikthtelev ${ }^{9}$. Ilkka Pohjalainen ${ }^{6}$. Sivaji Purushothman ${ }^{2}$. Zygmunt Patyk ${ }^{8}$. Moritz P. Reiter ${ }^{1,2,10}$. Ann-Kathrin Rink ${ }^{1}$. Sami Rinta-Antila ${ }^{6}$ - Anamaria Spataru 7,11 . Helmut Weick ${ }^{2}$. John S. Winfield ${ }^{2}$. Mikhail I. Yavor ${ }^{12}$ for the Super-FRS Experiment Collaboration

II. Physikalisches Institut, Justus-Liebig-Universität Gießen, 35392 Gießen, Germany

2 GSI Helmholtzzentrum für Schwerionenforschung GmbH, 64291 Darmstadt, Germany

3 Tel Aviv University, 6997801 Tel Aviv, Israel

4 Soreq Nuclear Research Center, 8180000 Yavne, Israel

5 Helsinki Institute of Physics, University of Helsinki, 00014 University of Helsinki, Finland

6 University of Jyväskylä, 40014 Jyväskylä, Finland

7 IFIN-HH/ELI-NP, 077126 Măgurele - Bucharest, Romania

8 National Centre for Nuclear Research, Hoza 69, 00-681 Warszawa, Poland

9 Institute for Energy Problems of Chemical Physics, RAS, 142432 Chernogolovka, Russia

10 TRIUMF, BC V6T 2A3 Vancouver, Canada

11 University Politehnica of Bucharest, Bucharest, Romania

12 Institute for Analytical Instrumentation, RAS, 190103 St. Petersburg, Russia 